\title{
Transportation, germs, culture: a dynamic graph model of 2019-nCoV spread
}

Xiaofei Yang ${ }^{1,2}$, Tun Xu $\mathrm{Xu}^{2,3}$, Peng Jia ${ }^{2,3}$, Han Xia ${ }^{3,4}$, Li Guo ${ }^{2,3,6}$, Kai Ye ${ }^{2,3,5,6, \dagger}$

${ }^{1}$ School of Computer Science and Technology, Faculty of Electronic and Information Engineering, Xi'an Jiaotong University, Xi'an, 710049 China.

${ }^{2}$ MOE Key Lab for Intelligent Networks \& Networks Security, Faculty of Electronic and Information Engineering, Xi'an Jiaotong University, Xi'an, 710049 China.

${ }^{3}$ School of Automation Science and Engineering, Faculty of Electronic and Information Engineering, Xi'an Jiaotong University, Xi'an, 710049 China.

${ }^{4}$ Hugobiotech Co., Ltd., Beijing 100000, China.

${ }^{5}$ Genome Institute, the First Affili-ated Hospital of Xi'an Jiaotong University, Xi'an, 710061 China.

${ }^{6}$ The School of Life Science and Technology, Xi'an Jiaotong University, Xi'an, 710049.China

†To whom correspondence should be addressed. E-mail: kaiye@xjtu.edu.cn

\section{Introduction}

Since the outbreak of 2019 novel coronavirus (2019-nCoV) at the hardest-hit city of Wuhan, the fast-moving spread has killed over three hundred people and infected more than ten thousands in China ${ }^{1}$. There are more than one hundred cases outside of China, affecting a dozen of countries globally². The genome sequence of 2019$\mathrm{nCoV}$ has been reported and fast diagnostic kits, effective treatment as well as preventive vaccines are rapidly being developed ${ }^{3}$. Initial fast-growing confirmed cases triggered lock-down of Wuhan as well as nearby cities in Hubei Province. Mathematical models have been proposed by scientists around the world to project the numbers of infected cases in the coming days ${ }^{4,5}$. However, major factors such as transportation and cultural customs have not been weighed enough. Our model is not set out for precise prediction of the number of infected cases, rather, it is meant for a glance of the dynamics under a public epidemic emergency situation and of different contributing 
factors. We hope that our model and simulation would provide more insights and perspective information to public health authorities around the globe for better informed prevention and containment solution.

\section{Methods}

In our model showed in Fig. 1A, we designed our dynamic graph model centered at transportation module as people take various transportation means to commute between places, considering that close-distance contacts during transportation are likely the most important factor aiding coronavirus transmission from infected to healthy ones. We built models accounting for taxi, bus, subway and walking with distinct capacities in the transportation module. We next placed stationary residential areas, public places and hospitals around the transportation module. Finally, we attached quarantine sites to hospitals. During each round of simulation, healthy or asymptomatic individual would start from one residential area, travel through the graph structure and returns to residence areas. Infected individuals would travel to hospitals after incubation period and remain at either hospital or quarantine sites if space allows. Otherwise, they will return to residential areas and seek medical care next day. The healthy individual becomes infected when he/she encounters infected ones during transportation or at any stationary areas. We performed the simulation beginning with one infected individual and observed the spread under various conditions.

\section{Results}

Although the major driver of pandemic spread are individuals with severe symptoms, data from Chinese cases in 2019-nCoV outbreak show signs of asymptomatic transmission. Thus, we first investigated the effects of asymptomatic transmission on viral epidemic. We assume that the capability of spreading increases linearly during incubation period and reaches maximum strength when symptom appears. We set the length of incubation period as 7 days and compared with symptomatic transmission. It is alarming that it took asymptomatic transmission half number of days to infect $50 \%$ of the population as with symptomatic transmission took (Fig. 1B). Whereas symptomatic individuals are much 
easier to identify and isolate from the general public, asymptomatic individuals transmit viruses unconsciously, exacerbating the disease epidemic. We next simulated various scenarios with varying lengths of contagious asymptomatic periods and observed that longer contagious incubation period speed up the transmission significantly, perhaps due to longer period of unconscious virus spreading (Fig. 1C).

A

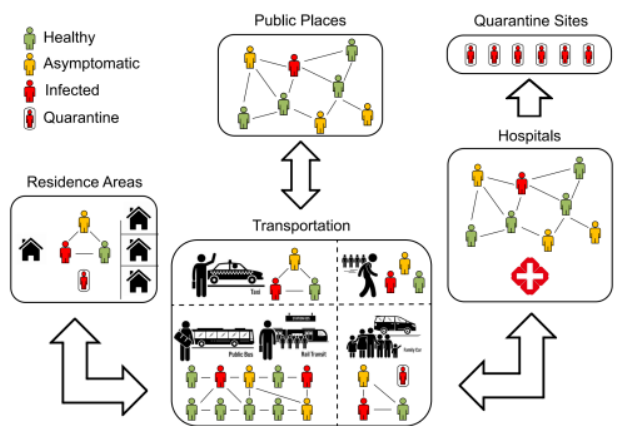

C

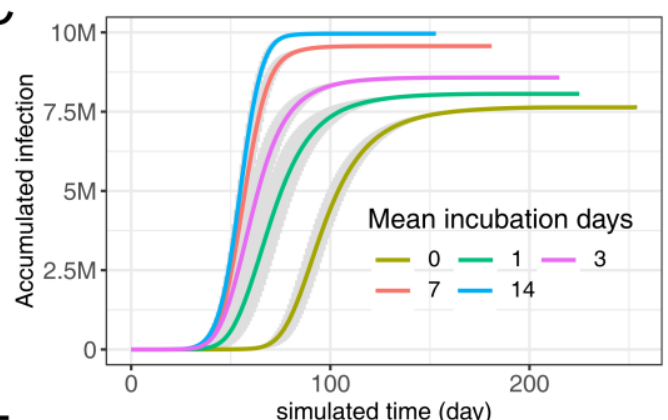

E

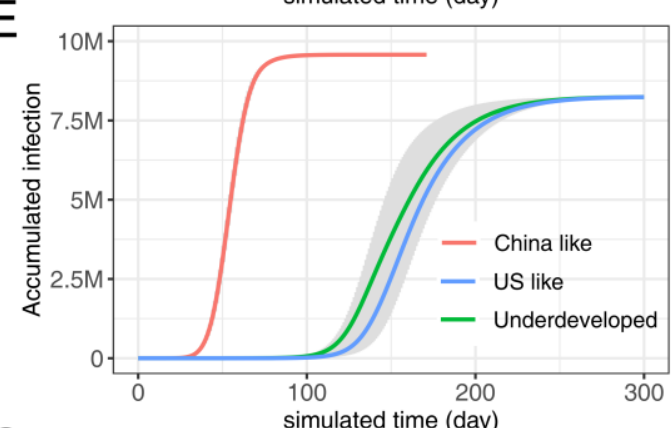

G

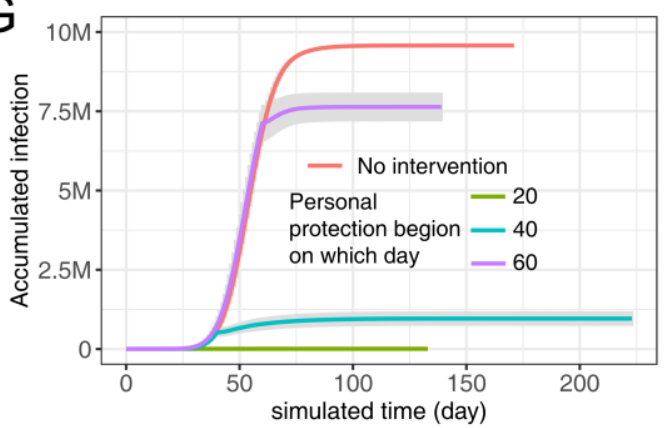

B

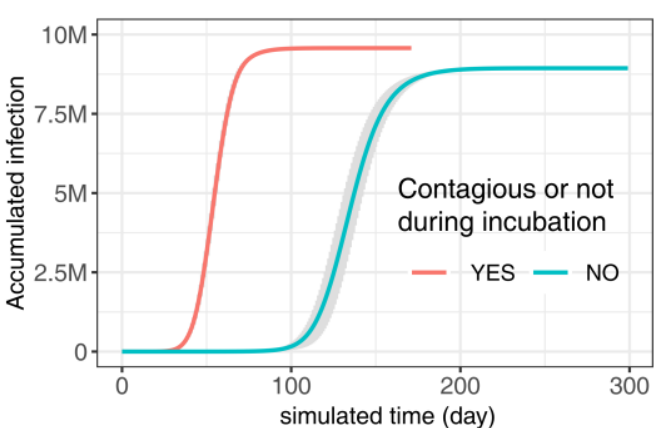

D

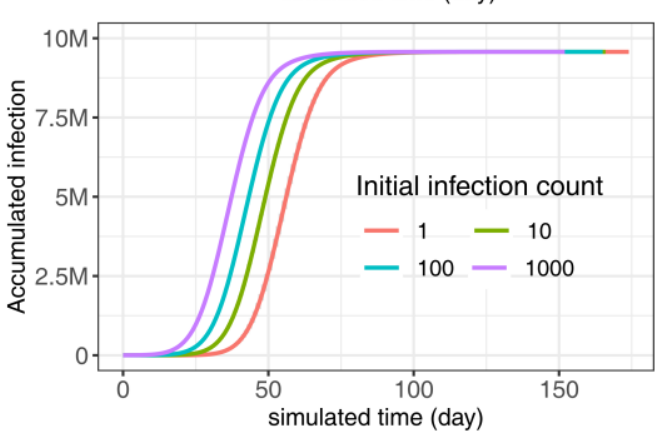

F

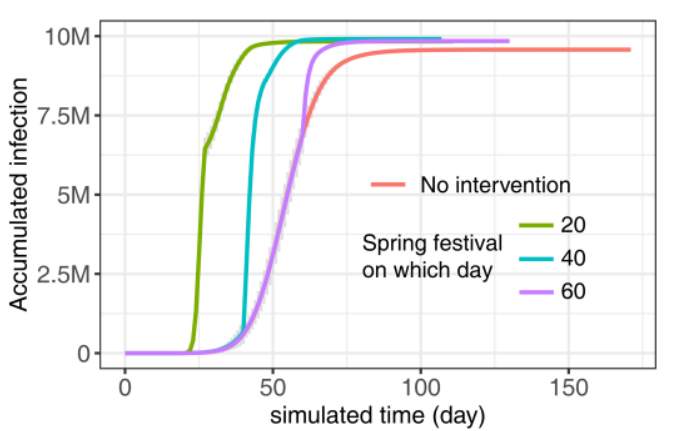

$\mathrm{H}$

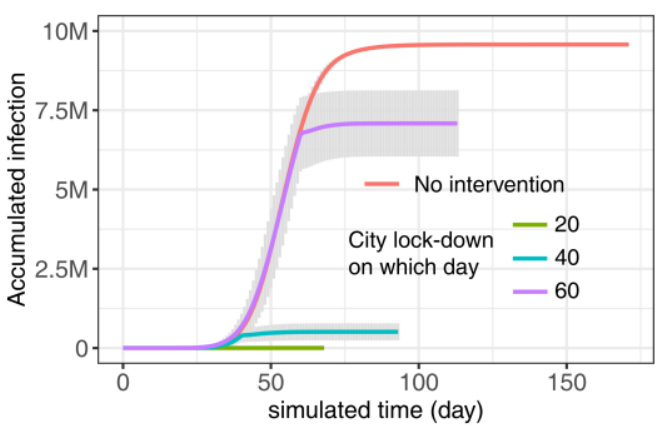

Fig. 1. The dynamic graph model and simulation results. In the simulation, we set the infection probability as $20 \%$, and the spreading probability as a linear function of infection time. 
dynamic graph model of contagious disease outbreak. B. Spreading patterns when asymptomatic transmission is present or absent. C. The impact of various lengths of asymptomatic periods on virus spread. D. The speed of viral spread given various imported cases in a city. E. Transportation affects viral spread. For US like simulation, we removed the public transportation; for underdeveloped simulation, we removed the public transportation and public places, as well as reducing the hospital capacity simultaneously. F. Social activities speed up viral spread. G. Wearing a mask and thorough hand sanitization significantly reduce the number of infected cases. We reduced $90 \%$ of both spreading and infection probabilities in personal protection simulation. $\mathbf{H}$. City lock-down halts transmission drastically.

Since the outbreak of 2019-nCoV, many cities have confirmed cases, mostly from Wuhan. We evaluated the effect of imported cases on the speed of spreading. As expected the more initial imported cases there are, the sooner $50 \%$ of the population are infected, as shown in Fig. 1D.

As transportation seems to be the major factor affecting the speed of spread, we simulated scenarios of typical cities around the world with different preferred transportation means. For example, combined public transportation like buses, subways and taxis are often the daily commute means in China while driving is the primary choice in US. We also considered a hypothetic underdeveloped city, where public transportation limited. We found that Chinese cities with advanced public transportation are particularly vulnerable to virus spreading while limited public transportation in an underdeveloped city halts virus transmission (Fig. 1E). In US where most families travel by driving, virus transmission is slowed down among families. This indicates that although convenient public transportation in modern cities provides enjoyable living styles, it unfortunately facilitates rapid spread of pandemic diseases.

We next evaluate how cultural social activities affect the spread of disease. The coronavirus epidemics outbroke around the traditional Chinese New Year, a holiday period when tens of millions of Chinese return home from workplace in major cities around the country for family reunion, also known as the Spring-festival Migration. It is estimated that over five million people departed from Wuhan during Spring-festival 
Migration right before the city lock-down. The infected individuals among them mostly returned to nearby small cities in the same province but still a number of them travelled to other Chinese cities and even overseas. Also, it is a Chinese tradition that relatives visit each other after the first day of Chinese New Year, which may continue for one week. We simulated the effect of family and relative during Spring festival and examined how would facilitate the spread of the virus. As shown in Fig. 1F, earlier gatherings would stimulate wide spread of virus. Fortunately, Chinese civilians were warned about the severe consequence of social gathering during 2019-nCoV outbreak and as a result most of them stayed at home to avoid contact with others for at least a dozen of days.

It is not unusual to require civilians to wear masks in public and to wash hands thoroughly in order to halt virus epidemic, but to lock-down major cities of the size like Wuhan requires considerate resources. We found that both personal protection (Fig. 1G) and city lock-down (Fig. 1H) halts transmission of virus immediately and the earlier installation of those orders the lower maximum number of infected cases. Although lockdown of the cities seems a radical administrative action, likely having a major impact on economy, the actions so far taken by Chinese authorities have certainly minimized the spreading of the virus globally.

\section{Discussion and conclusion}

Taken together, we conclude that actions taken by authorities in China such as locking down cities and strict requirement of personal protection and sanitization have reduced the viral spread significantly. Efficient and effective communication and well-educated general public are the keys to contain this pandemic spread. We recommend that CDC (center of disease control) or equivalent authorities in all countries shall pay close attention to possible asymptomatic transmission as dramatic changes in quarantine protocol are necessary if data supports its possibility.

Code Availability: https://github.com/xjtu-omics/2019-nCoV_graph_model 


\section{Funding}

This study was supported by the National Science Foundation of China (grand NO. 61702406 and 31671372, 31701739), the National Key R\&D Program of China (grand NO. 2018YFC0910400, 2017YFC0907500), the National Science and Technology Major Project of China (grand NO. 2018ZX10302205), and the "World-Class Universities and the Characteristic Development Guidance Funds for the Central Universities".

\section{Reference}

1. Update on the novel coronavirus pneumonia outbreak 2020. at http://www.nhc.gov.cn/xcs/yafkdt/202001/c5da49c4c5bf4bcfb320ec2036480627.shtml.)

2. CDC Telebriefing: Update on 2019 Novel Coronavirus (2019-nCoV). Second Travelrelated Case of 2019 Novel Coronavirus Detected in United States. 2020. at https://www.cdc.gov/media/releases/2020/p0124-second-travel-coronavirus.html.)

3. Lu R, Zhao X, Li J, et al. Genomic characterisation and epidemiology of 2019 novel coronavirus: implications for virus origins and receptor binding. The Lancet.

4. Read JM, Bridgen JR, Cummings DA, Ho A, Jewell CP. Novel coronavirus 2019-nCoV: early estimation of epidemiological parameters and epidemic predictions. medRxiv 2020.

5. Li Q, Guan X, Wu P, et al. Early Transmission Dynamics in Wuhan, China, of Novel Coronavirus-Infected Pneumonia. New England Journal of Medicine 2020. 